\title{
Increased Proliferation as Independent Predictor of Disease Recurrence in Initial Stage pTa Urothelial Bladder Cancer
}

\author{
Johannes Breyer ${ }^{\mathrm{a}, 1, *}$, Sanzhar Shalekenov ${ }^{\mathrm{a}, 1}$, Atiqullah Aziz ${ }^{\mathrm{b}}$, Bastiaan W.G. van Rhijn ${ }^{\mathrm{a}, \mathrm{c}}$, \\ Johannes Bründl $^{\mathrm{a}}$, Eva Lausenmeyer ${ }^{\mathrm{a}}$, Julius Schäfer ${ }^{\mathrm{a}}$, Stefan Denzinger ${ }^{\mathrm{a}}$, Christian Giedl ${ }^{\mathrm{d}}$, \\ Maximilian Burger ${ }^{\mathrm{a}}$, Arndt Hartmann ${ }^{\mathrm{e}}$, Matthias Evert ${ }^{\mathrm{d}}$ and Wolfgang Otto ${ }^{\mathrm{a}}$ \\ ${ }^{a}$ Department of Urology, Caritas St. Josef Medical Center, University of Regensburg, Regensburg, Germany \\ ${ }^{\mathrm{b}}$ Department of Urology, University of Hamburg, Hamburg, Germany \\ ${ }^{\mathrm{c}}$ Department of Surgical Oncology (Urology), Netherlands Cancer Institute-Antoni van Leeuwenhoek Hospital, \\ Amsterdam, The Netherlands \\ ${ }^{\mathrm{d}}$ Institute of Pathology, University of Regensburg, Regensburg, Germany \\ ${ }^{\mathrm{e}}$ Institute of Pathology, University of Erlangen-Nuremberg, Erlangen, Germany
}

\begin{abstract}
.
Objectives: To investigate the predictive impact of the proliferation biomarker Ki-67 on the clinical course of patients with initial stage pTa urothelial carcinoma of the bladder (UCB).

Methods: We retrospectively analyzed all patients treated by transurethral resection of bladder tumors (TUR-B) for UCB between 1992-2004 in a single-center. Disease recurrence ( $\geq$ pTa UCB) and absent tumor in histopathology, assessed by TUR-B with a non-malignant result for endoscopic suspect bladder lesion displayed endpoints. Immunohistochemical (IHC) analysis of formalin-fixed and paraffin-embedded tissue blocks was performed with an immunostainer using a primary antibody for Ki-67. Semiquantitative evaluation of Ki-67 was performed by three reviewers. Increased proliferation was defined with a cut-off value of $\geq 50 \%$. Uni- and multivariable binary regression analyses were applied to address prediction of disease recurrence.

Results: 215 patients ( $84 \%$ male, median age 69 years at first diagnosis) were evaluable and included to the study. 89 patients stayed disease-free (41\%), 126 patients showed recurrence (59\%). Recurrence rates of patients with Ki-67 expression $<10 \%$, $10-24 \%, 25-49 \%$ and $\geq 50 \%$ were $14.8 \%$ vs. $30.8 \%$ vs. $63.9 \%$ and $80.7 \%$, respectively $(p<0.001)$. In Kaplan-Meier analysis patients with increased proliferation $\geq 50 \%$ showed a statistically significant worse 10 -year recurrence-free survival (19\% vs. $57 \%, p<0.001)$. Multivariable regression analysis revealed instillation treatment $(p=0.001)$ and high proliferation of Ki-67 $(p<0.001)$ to be independent predictors of recurrence in stage pTa UCB.

Conclusions: High proliferation with Ki-67 expression $\geq 50 \%$ was strongly associated with worse recurrence-free survival in patients with initial stage pTa UCB. Stage pTa UCB patients with increased Ki-67 expression should undergo a strictly follow-up regime comparable to stage pT1 bladder carcinoma, while at least patients with Ki-67 expression $<10 \%$ might be feasible for more liberate follow-up regime after evaluation of our data in randomized, prospective and multicenter studies.
\end{abstract}

Keywords: Immunohistochemistry, Ki-67, recurrence, urothelial bladder cancer

\footnotetext{
${ }^{1}$ equal contribution.

${ }^{*}$ Correspondence to: Johannes Breyer, Caritas St. Josef Medical Center, Department of Urology, University of Regens-
}

burg, Landshuter Str. 65, 93053 Regensburg, Germany. Tel.: +49 941782 3534; Fax: +49 941782 3515; E-mail: hannes.breyer@ gmx.de 


\section{INTRODUCTION}

Urothelial carcinoma of the bladder (UCB) is the fifth most common cancer worldwide. More than $75 \%$ of UCB patients present with a non-muscle-invasive disease at initial diagnosis [1]. The majority of these cases can be managed with transurethral resection (TUR) and intravesical therapy alone [2]. Nevertheless, roughly $30 \%$ of patients with high-grade non muscle-invasive UCB will eventually suffer from disease progression to muscle-invasive UCB and consequently demand surgical management with radical cystectomy (RC) and additional perioperative therapy in some cases [3].

The incidence of UCB and the need of a lifetime follow-up due to the high recurrence rates of up to $75 \%$, is associated with very high costs for our health care system. Thus, an improvement in diagnostic, therapeutic, and follow-up strategies is required. Stratifying patients in distinct risk groups has become a cornerstone in outcome prediction for UCB. The European Organization for Research and Treatment of Cancer (EORTC) calculator displays an established tool for calculating individual patient's risk of disease recurrence and progression after transurethral resection of bladder tumors (TURB) [4]. Based on the EORTC calculator and other well-established UCB predictors, patients can be stratified into three different risk groups according to current guidelines [3]. Nevertheless, the probabilities of disease recurrence and progression in patients with UCB are varying significantly, especially in pTa UCB which tend to recur frequently [5]. By definition, pTa describes a non-invasive papillary carcinoma [6]. Although this tumor stage seems to be of lower malignant nature, patients staged pTa are more likely to have a fivefold higher risk for diseasespecific mortality than the general population [7]. Nevertheless, outcome prediction in this heterogeneous cohort of patients with initial diagnosis of $\mathrm{pTa}$ UCB still remains challenging.

Most of the reports currently available regarding immunohistochemical predictive markers in UCB address invasive disease. High expression of the proliferation marker Ki-67 is associated with more aggressive tumor biology and thus with an adverse outcome in pT1 UCB [8-10]. We hypothesized that this immunohistochemical marker may also have a prognostic value in patients with pTa UCB. Thus the aim of the current study was to analyze the predictive impact of $\mathrm{Ki}-67$ on tumor recurrence within a single-center series of initially diagnosed pTa UCB after TUR-B.

\section{METHODS}

\section{Study population and treatment}

This was an institutional review board approved study. All of the patients were extracted from a singlecenter database comprising 1366 patients with pTa UCB who underwent TUR-B according to guidelines recommendations between 1992 and 2004. Only patients with either no further tumor or recurrent disease by histopathological evaluation in repetitive TUR-B were included in the study. Patients, who probably were transurethral resected during followup regime, but not at our hospital, could not be included into the study as did patients without any clinical occurrence of disease if not histopathologically proven. Patients who could not be followed-up precisely for at least 72 months were also excluded from the study. Thus, a total of 233 patients with initial diagnosis of pTa UCB after TUR-B and respective second TUR-B, where appropriate, were eligible for final analysis. After TUR-B and histopathological diagnosis of pTa UCB, patients underwent a second TUR-B due to presence of a grade 3 tumor (WHO 1973), multifocal tumors, associated carcinoma in situ (Cis), tumors with diameter $>3 \mathrm{~cm}$ or lack of muscle in the specimen [3]. Immediate instillation of chemotherapy was not administered routinely in this period. However, various regimes were individually applied when high grade tumor or associated carcinoma in situ (Bacillus CalmetteGuérin) and/or multifocal tumors (Mitomycin C) were present, respectively. Please refer to Table 1.

\section{Pathologic evaluation}

All surgical specimens were processed according to standard pathologic procedures. Genitourinary pathologists assigned tumor grade according to the 1973 World Health Organization (WHO) grading system. Pathologic stage was reassigned according to the 2009 American Joint Committee on Cancer TNM staging system [6].

\section{Immunohistochemistry: Assessment and evaluation}

Four-micrometer sections were cut and mounted on poly-L-lysine coated glass slides from formalin- 
Table 1

Patient characteristics

\begin{tabular}{|c|c|c|c|}
\hline Parameter & $\begin{array}{c}\text { Disease-free } \\
\text { patients }(n=89)\end{array}$ & $\begin{array}{l}\text { Patients with reccurent } \\
\text { disease }(n=126)\end{array}$ & $p$ value \\
\hline $\begin{array}{l}\text { Age (median } 69 \text { years, IQR } 61-76 \text { years; } \\
\text { share of patients } \geq 70 \text { years compared) }\end{array}$ & $38(42.7 \%)$ & $71(56.3 \%)$ & $p=0.033$ \\
\hline \multicolumn{4}{|l|}{ Gender } \\
\hline male & $73(82.0 \%)$ & $108(85.7 \%)$ & \multirow[t]{2}{*}{$p=0.293$} \\
\hline female & $16(18.0 \%)$ & $18(14.3 \%)$ & \\
\hline \multicolumn{4}{|l|}{ WHO Grading 1973} \\
\hline G1 & $38(42.7 \%)$ & $65(51.6 \%)$ & \multirow[t]{3}{*}{$p=0.352$} \\
\hline $\mathrm{G} 2$ & $43(48.3 \%)$ & $54(42.9 \%)$ & \\
\hline G3 & $8(9.0 \%)$ & $7(5.5 \%)$ & \\
\hline Associated CIS & $8(9.0 \%)$ & $4(3.2 \%)$ & $p=0.064$ \\
\hline \multicolumn{4}{|l|}{ Focality } \\
\hline unifocality & $63(70.8 \%)$ & $85(67.5 \%)$ & \multirow[t]{2}{*}{$p=0.357$} \\
\hline multifocality & $26(29.2 \%)$ & $41(32.5 \%)$ & \\
\hline \multicolumn{4}{|l|}{ Tumor size } \\
\hline Tumor $<3 \mathrm{~cm}$ & $83(93.3 \%)$ & $115(91.3 \%)$ & \multirow[t]{2}{*}{$p=0.396$} \\
\hline Tumor $\geq 3 \mathrm{~cm}$ & $6(6.7 \%)$ & $11(8.7 \%)$ & \\
\hline \multicolumn{4}{|l|}{ Symptoms } \\
\hline Hematuria & $34(38.2 \%)$ & $38(30.2 \%)$ & \multirow[t]{3}{*}{$p=0.270$} \\
\hline Dysuria & $6(6.7 \%)$ & $6(4.7 \%)$ & \\
\hline None & $49(55.1 \%)$ & $82(65.1 \%)$ & \\
\hline \multicolumn{4}{|l|}{ Instillation therapy } \\
\hline None & $49(55.1 \%)$ & $98(77.8 \%)$ & \multirow[t]{3}{*}{$p=0.070$} \\
\hline Mitomycin C & $30(33.7 \%)$ & $19(15.1 \%)$ & \\
\hline $\mathrm{BCG}$ & $10(11.2 \%)$ & $9(7.1 \%)$ & \\
\hline
\end{tabular}

Abbreviations: BCG: Bacillus Calmette-Guérin, CIS: Carcinoma in situ; IQR: interquartile range.

fixed and paraffin-embedded tissue blocks. IHC analysis was carried out in a Ventana ${ }^{\odot}$ BenchMark IHC Full System immunostainer (Roche Diagnostics, Mannheim, Germany) using the avidin-biotin peroxidase method with diaminobenzidine as a chromatogen according to the manufacturer's instructions. The primary antibody of Ki-67 was used as shown in Fig. 1. Marker expression was visualized with a Primo Star microscope (Carl Zeiss Microimaging, Jena, Germany) under 400-fold magnification. Evaluation of UCB immunostaining was performed independently by three reviewers without knowledge of clinical and follow-up data. Ki-67 was assessed in a 4-tier system $(<10 \%, 10-24 \%, 25-49 \%, \geq 50 \%)$ and overexpression was defined if $\geq 50 \%$ of urothelial tumor cells stained positive. In case of interobserver variation was solved by majority decision

\section{Follow-up treatment}

Patients were generally followed every 3-6 months for the first 2 years after TUR-B, biannually up to 5 years, and annually thereafter. Follow-up included history, physical examination, urinary cytology, cystoscopy, and biopsy of suspicious lesions according to guidelines of the European Association of Urology (EAU). Radiographic evaluation of the upper urinary tract was generally done at diagnosis and in case of disease recurrence or suspicion, which were positive cytology or suspicious ultrasound of the upper urinary tract during follow-up. Disease recurrence was defined as first histologically documented tumor relapse in the bladder or prostatic urethra, regardless of tumor stage and displayed our end-point. When disease recurrence was registered, TUR-B was performed. Only patients with at least one further suspect lesion during follow-up that was biopsied or transurethrally resected and proven to be nonmalignant, e.g. cystitis, by histopathological means were classified disease-free and included in the study. All patients with proven recurrence and patients with a pathologically proven disease-free follow-up of at least 72 months were included.

\section{Statistical analysis}

The Kolmogorov-Smirnov test was applied to investigate the normal distribution of continuous variables. Continuous variables are presented as mean with standard deviations (SD) or as median with interquartile ranges (IQR), depending on the type of distribution. Comparison between categorical variables was performed using Fisher's exact and chi-square-test. The Wilcoxon-rank-sum-test 

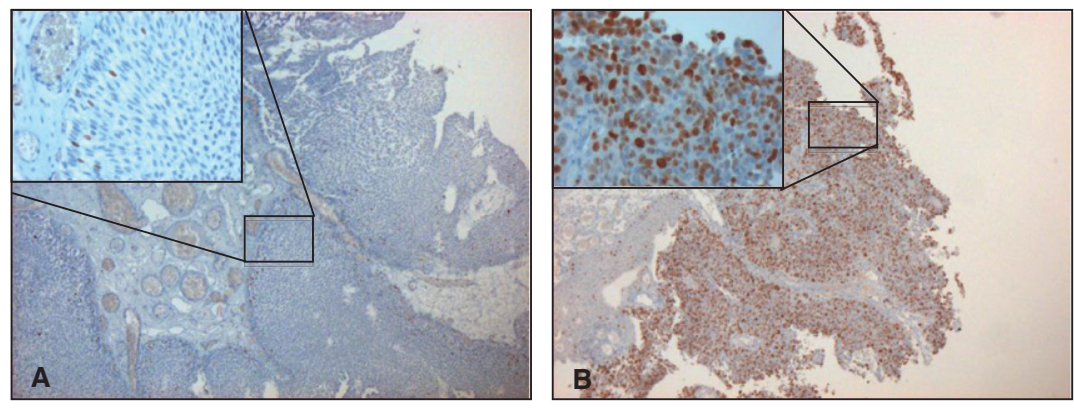

\begin{tabular}{|c|c|c|c|}
\hline $\begin{array}{l}\text { C } \\
\text { Ki-67 } \\
\text { expression }\end{array}$ & $\begin{array}{c}\text { disease-free } \\
\text { patients } \\
(n=89)\end{array}$ & $\begin{array}{c}\text { recurrent } \\
\text { patients } \\
(n=126)\end{array}$ & p value \\
\hline$<10 \%$ & $23(25.8 \%)$ & $3.2 \%)$ & \multirow{4}{*}{$<0.001$} \\
\hline$<25 \%$ & $50(56.1 \%)$ & $16(12.7 \%)$ & \\
\hline$<50 \%$ & $72(80.8 \%)$ & 55 ( $43.7 \%)$ & \\
\hline$\geq 50 \%$ & $89(100.0 \%)$ & $126(100.0 \%)$ & \\
\hline
\end{tabular}

Fig. 1. Examples of low (here $<10 \%$, A) and strong (here $\geq 50 \%$, B) staining of Ki-67 under 5 fold and 40 fold (left above) magnification. Correlation of Ki-67 expression with disease-free and recurrent patients by cumulative numbers and rates (C).

(Mann-Whitney-U; two categories) and the KruskalWallis-test (three or more categories) was applied for not normally distributed variables. KaplanMeier analysis was used to assess impact of the immunohistochemical parameter for recurrence-free survival (RFS). Multivariable binary logistic regression models evaluated the impact of the established clinico-pathologic features and the marker Ki-67 on disease recurrence. All $\mathrm{p}$ values were two-sided, and statistical significance was defined as $p<0.05$. Statistical analyses were performed using IBM SPSS Statistics, version 23.0 (SPSS Inc, Chicago, IL).

\section{RESULTS}

\section{Patient characteristics}

The number of TUR-Bs in the follow-up period varied between one and ten between the single patients. Tumor specimens of 215 patients $(92 \%)$ for Ki-67 were suitable for immunohistochemical evaluation. While 89 patients $(41 \%)$ never showed malignant findings in their follow-up TUR-Bs, 126 patients $(59 \%)$ had at least one urothelial carcinoma recurrence, of which 27 patients showed progress to at least pT1 bladder cancer. Table 1 gives detailed information about the descriptive characteristics of our study population. Due to high recurrence rate median follow-up was 36 months with an IQR of 13 to 125 months with a maximum follow-up of 199 months.

\section{Association of Ki-67 expression and histopathological parameters for UCB recurrence and correlation of Ki-67 expression with clinical and histopathological features}

Of studied clinicopathological parameters including instillation treatment only higher age $\geq 70$ years (56\% vs. $43 \%$ in non-recurrent disease, $p=0.033$ ) was associated with disease recurrence (Table 1). Correlation of Ki-67 expression with various clinical and histopathological features including instillation treatment showed no statistically significant associations (Table 2).

\section{Kaplan-Meier analysis of Ki-67 expression for recurrence-free survival}

Recurrence rates of patients with Ki-67 expression $<10 \%, 10-24 \%, 25-49 \%$ and $\geq 50 \%$ were $14.8 \%$ vs. $30.8 \%$ vs. $63.9 \%$ and $80.7 \%$, respectively $(p<0.001$, Fig. 2A). Kaplan-Meier analysis showed a statistically significantly worse 10-year RFS for patients with Ki-67 overexpression $\geq 50 \%$ (19\% vs. $57 \%$, $p<0.001$, Fig. 2B).

\section{Multivariate regression analysis regarding disease recurrence after initial pTa UCB}

In univariate regression analysis $\mathrm{Ki}-67$ expression $\geq 50 \%$ displayed the most predictive factor for recurrent disease (OR 2.632, CI: 1.845-3.753, $p<0.001$ ), followed by instillation treatment (OR $0.504, \mathrm{CI}$ : 
Table 2

Correlation of Ki-67 expression with established clinical and histopathological features

\begin{tabular}{|c|c|c|c|}
\hline Parameter & $\begin{array}{l}\text { Ki-67 expression } \\
<50 \%(n=127)\end{array}$ & $\begin{array}{c}\text { Ki-67 expression } \\
\geq 50 \%(n=88)\end{array}$ & $p$ value \\
\hline \multicolumn{4}{|l|}{$\overline{\text { Age }}$} \\
\hline age $\leq 69$ years & $68(53.5 \%)$ & $38(43.2 \%)$ & \multirow[t]{2}{*}{$p=0.088$} \\
\hline age $\geq 70$ years & $59(46.5 \%)$ & $50(56.8 \%)$ & \\
\hline \multicolumn{4}{|l|}{ Gender } \\
\hline male & $108(85.0 \%)$ & $73(83.0 \%)$ & \multirow[t]{2}{*}{$p=0.409$} \\
\hline female & $19(15.0 \%)$ & $15(17.0 \%)$ & \\
\hline \multicolumn{4}{|c|}{ WHO Grading 1973} \\
\hline G1 & $63(49.7 \%)$ & $40(45.5 \%)$ & \multirow[t]{3}{*}{$p=0.129$} \\
\hline $\mathrm{G} 2$ & $52(40.9 \%)$ & $45(51.1 \%)$ & \\
\hline G3 & $12(9.4 \%)$ & $3(3.4 \%)$ & \\
\hline Associated CIS & $9(4.2 \%)$ & $3(3.4 \%)$ & $p=0.199$ \\
\hline \multicolumn{4}{|l|}{ Focality } \\
\hline unifocality & $86(67.7 \%)$ & $62(70.5 \%)$ & \multirow[t]{2}{*}{$p=0.392$} \\
\hline multifocality & $41(32.3 \%)$ & $26(29.5 \%)$ & \\
\hline \multicolumn{4}{|l|}{ Tumor size } \\
\hline Tumor $<3 \mathrm{~cm}$ & $117(92.1 \%)$ & $81(92.0 \%)$ & \multirow[t]{2}{*}{$p=0.588$} \\
\hline Tumor $\geq 3 \mathrm{~cm}$ & $10(7.9 \%)$ & $7(8.0 \%)$ & \\
\hline \multicolumn{4}{|l|}{ Instillation therapy } \\
\hline None & $89(70.1 \%)$ & $58(65.9 \%)$ & \multirow[t]{3}{*}{$p=0.546$} \\
\hline Mitomycin C & $29(22.8 \%)$ & $20(22.7 \%)$ & \\
\hline $\mathrm{BCG}$ & $9(7.1 \%)$ & $10(11.4 \%)$ & \\
\hline
\end{tabular}

Abbreviations: BCG: Bacillus Calmette-Guérin, CIS: Carcinoma in situ.
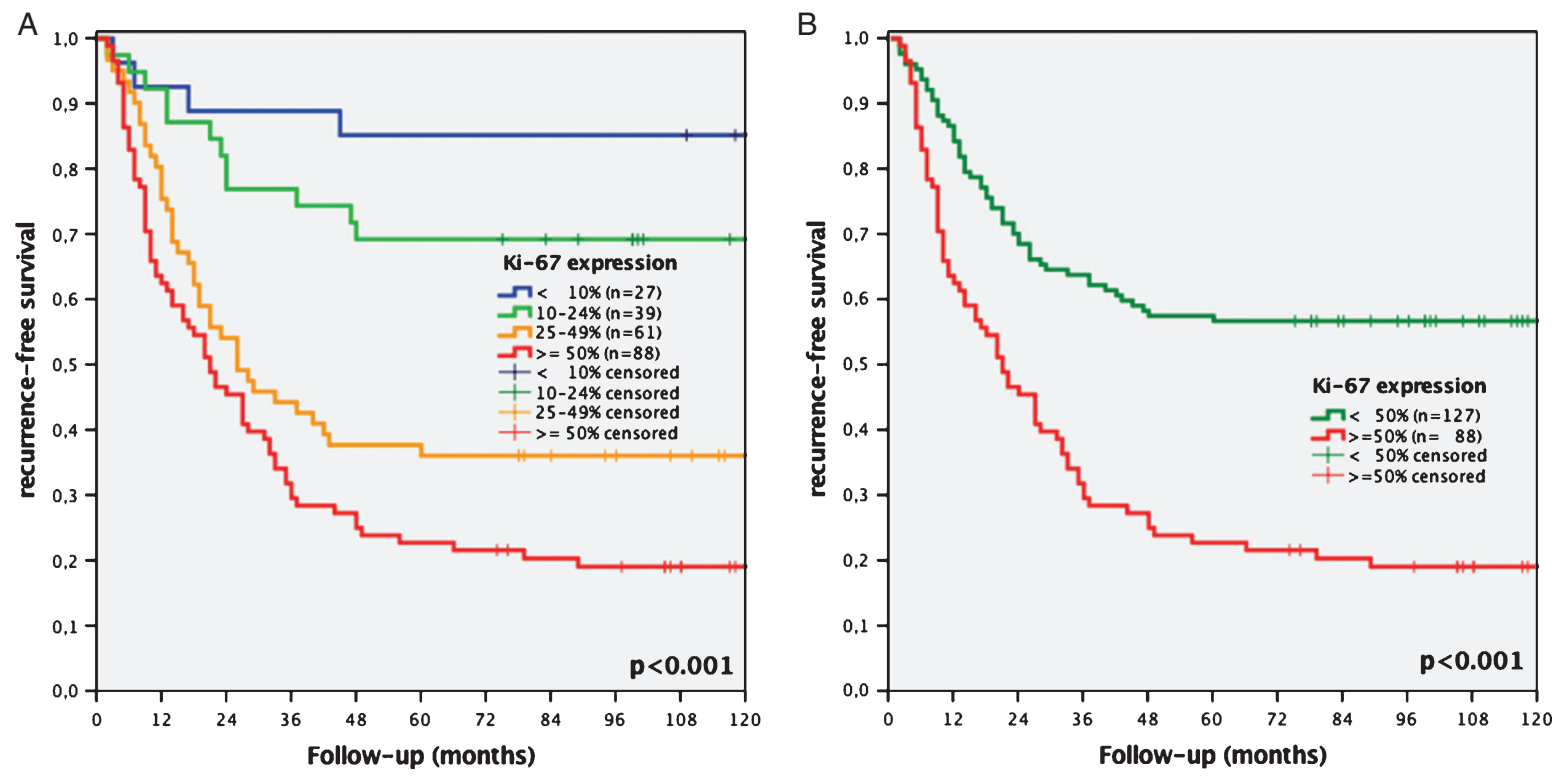

Fig. 2. Kaplan-Meier analyses of Ki-67 expression of a 4 -tier ( $<10 \%$ vs. $10-24 \%$ vs. $25-49 \%$ vs. $\geq 50 \%$, A) and 2 -tier cut-off system ( $<50 \%$ vs. $\geq 50 \%, \mathrm{~B}$ ) regarding recurrence-free survival concerning all stages of recurrence of initial stage pTa bladder cancer patients.

$0.330-0.767, p=0.001)$ and by higher age (OR 1.024, CI: $1.007-1.042, p=0.006$, Table 3a). In multivariate regression analysis of these markers overexpression of Ki-67 (HR 2.553, CI: 1.779-3.664, $p<0.001$ ) remained of independent predictive value for recurrence as well as instillation treatment (HR 0.484, CI: $0.317-0.738, p=0.001$, Table $3 b$ ).

\section{DISCUSSION}

The clinical relevance of a prognostic biomarker requires verification that the use of a biomarker can index patients into distinct subgroups with different recommendations regarding disease management before application in daily clinical routine [11]. 
Table 3

Univariate (a) and multivariate (b) binary regression analysis of clinicopathological parameters and Ki-67 expression predicting recurrence of initial stage pTa urothelial carcinoma of the bladder

a)

\begin{tabular}{lcc}
\hline Parameter & $\begin{array}{c}\text { Odds ratio } \\
\text { (95\% Confidence Interval) }\end{array}$ & $p$ value \\
\hline Gender female vs. male & $0.814(0.494-1.341)$ & 0.420 \\
Age per year & $\mathbf{1 . 0 2 4}(\mathbf{1 . 0 0 7}-\mathbf{1 . 0 4 2})$ & $\mathbf{0 . 0 0 6}$ \\
WHO Grading 1973 G3 vs. G1/2 & $0.821(0.383-1.760)$ & 0.613 \\
Associated CIS & $0.497(0.184-1.346)$ & 0.169 \\
Multifocality & $1.126(0.776-1.635)$ & 0.532 \\
Tumor size $\geq 3$ vs. $<3 \mathrm{~cm}$ & $1.258(0.678-2.336)$ & 0.467 \\
Instillation therapy yes vs. no & $\mathbf{0 . 5 0 4}(\mathbf{0 . 3 3 0 - 0 . 7 6 7 )}$ & $\mathbf{0 . 0 0 1}$ \\
Ki-67 expression $\geq 50 \%$ vs. $<50 \%$ & $\mathbf{2 . 6 3 2}(\mathbf{1 . 8 4 5}-\mathbf{3 . 7 5 3})$ & $<\mathbf{0 . 0 0 1}$ \\
\hline
\end{tabular}

Abbreviations: CI: confidence interval, CIS: carcinoma in situ; WHO: World Health Organization.

b)

\begin{tabular}{lcc}
\hline Parameter & $\begin{array}{c}\text { Hazard ratio } \\
(95 \% \text { Confidence Interval })\end{array}$ & $p$ value \\
\hline Age per year & $1.015(0.997-1.034)$ & 0.093 \\
Instillation therapy yes vs. no & $\mathbf{0 . 4 8 4}(\mathbf{0 . 3 1 7 - 0 . 7 3 8 )}$ & $\mathbf{0 . 0 0 1}$ \\
Ki-67 expression $\geq 50 \%$ vs. $<50 \%$ & $\mathbf{2 . 5 5 3}(\mathbf{1 . 7 7 9 - 3 . 6 6 4 )}$ & $<\mathbf{0 . 0 0 1}$ \\
\hline
\end{tabular}

Abbreviations: CI: confidence interval. Bold value significant is $p<0.05$.

A recent retrospective series of pT1 UCB demonstrated that $\mathrm{Ki}-67$ is related with features of biologically aggressive UCB, as well as important clinical outcomes, i.e. disease recurrence, progression and disease-specific survival [8-10]. The aim of the present study was to assess whether this marker can identify patients with pTa UCB after first TUR-B with high risk for recurrence. An accurate prediction of recurrence at TUR-B would allow identification of the patients with pTa UCB who would benefit from a closer follow-up and eventually an intravesical chemotherapy instillation regime. We showed in the present study that beside the established features of outcome prediction, like age and instillation treatment, tumor cell proliferation independently predicted disease recurrence. Nevertheless, when implementing a biomarker in daily clinical routine, it is pivotal that it adds meaningful information to already known clinically data. Ki-67 is a very useful marker of cell proliferation and is routinely used in many different tumor types like breast cancer or neuroendocrine tumors to predict prognosis and stratify therapy [8]. The antibody MIB-1 detects the nuclear antigen Ki-67, expressed in nuclei during the G1, S, and G2 but not the G0 phase [8].

In UCB, the Ki-67 index has been described to correlate significantly with tumor recurrence, progression and survival in non muscle-invasive
UCB [11-14] and in muscle-invasive UCB [15-17], respectively. The findings of van Rhijn et al. incorporating a molecular grading method for UCB combining FGFR 3 mutations and Ki-67 proliferation index showed significant associations with recurrence, progression, and disease-specific survival in all stages of UCB [18]. The study group validated their findings and demonstrated a prognostic role of molecular grade in primary non muscle-invasive UCB [19]. Shariat et al. previously presented their findings addressing the predictive capacity of biomarkers of upstaging at radical cystectomy. Within a panel of 5 biomarkers including Cyclin E, p53, p21, p27 and $\mathrm{Ki}-67$ of high grade tumors at last TUR-B before $\mathrm{RC}$, only p27 was significantly associated with a Tstage $(p=0.020)$ and $\mathrm{T}$ - and/or $\mathrm{N}$-stage $(p=0.040)$ upstaging. However, Ki-67 showed a strong tendency towards, but did not reach statistically significance to $\mathrm{T}$ - and/or $\mathrm{N}$-stage upstaging $(p=0.051)$ [11]. In our study no correlation of grading and other clinicopathological parameters with recurrence could be found, perhaps due to low number of pTaG3, associated Cis and tumor size $\geq 3 \mathrm{~cm}$.

Disease recurrence in pTa UCB is frequently observed and thus underscores the heterogeneous nature of the disease at this stage. Besides aberrant expression of $\mathrm{Ki}-67$, we could confirm that lack of instillation treatment significantly impacted on disease recurrence, which is in line with other findings 
[20, 21]. A total of $126(59 \%)$ patients within the present study on initially pTa UCB presented with disease recurrence during follow-up. The recurrence rates in our study are in line with another study by Holmäng et al., in which 143 (58\%) patients with pTa experienced disease recurrence [22]. Rieken et al. previously published their data including more than 1400 patients with pTa G1 UCB, of which $56 \%$ were recurrence-free after 5 years within a median follow-up of 67.2 months [20]. Linton et al. presented their data incorporating 699 patients with primary TaG1 UCB, of which $28.5 \%$ had a disease recurrence at a median follow-up of 13.5 months [7]. There was no study until now that analysed Ki-67 in a solely stage pTa UCB cohort. Holmäng et al. had analysed a cohort of papillomas, PUNLMP and pTa $\mathrm{UCB}$, Gontero et al., Quintero et al. and Blandamura et al. collectives of mixed stages pTa and pT1 UCB [23-26]. Only Quintero et al. showed favourable outcome of patients with higher Ki-67 expression, the other studies were in line with our results, showing worse recurrence rates of patients with overexpressed Ki-67 staining.

Our study is not devoid of limitations. There are issues associated to the reliability of immunohistochemical biomarker assessment, which are highly dependent on a range of poorly controlled variables, including choice of antibody, antibody dilution, fixation techniques, and inconsistency in specimen handling [11]. We aimed to impair the number of variables in the immunohistochemical techniques by using highly standardized protocols, including established staining protocols. Our study was conducted in a retrospective fashion, thus including all limitations inherent to the retrospective nature of the study.

To conclude, patients with pTa UCB at initial TUR-B have a high risk of recurrence. While established clinico-pathological features did not show prognostic impact, high proliferation detected by expression of the biomarker Ki-67 in the TUR-B specimen improves the prediction of recurrence. Further biomarker panels addressing outcome in pTa UCB should therefore incorporate Ki-67. Further studies in this issue, ideally in a prospective multicenter fashion are required.

\section{ACKNOWLEDGMENTS}

The authors thank Mrs. Stefanie Götz for her excellent assistance in immunohistochemical procedures.

\section{DISCLOSURE OF INTEREST}

There is no substantial direct or indirect commercial financial incentive associated with publishing the article.

\section{REFERENCES}

[1] Burger M, Catto JW, Dalbagni G, et al. Epidemiology and risk factors of urothelial bladder cancer. Eur Urol 2013;63:234-41.

[2] Siegel R, Naishadham D, Jemal A. Cancer statistics, 2013. CA Cancer J Clin 2013;63:11-30.

[3] Babjuk M, Burger M, Zigeuner R, et al. EAU guidelines on non-muscle-invasive urothelial carcinoma of the bladder: Update 2013. Eur Urol 2013;64:639-53.

[4] Sylvester RJ, van der Meijden APM, Oosterlinck W, et al. Predicting recurrence and progression in individual patients with stage Ta T1 bladder cancer using EORTC risk tables: A combined analysis of 2596 patients from seven EORTC trials. Eur Urol 2006;49:466-77, discussion 475-7.

[5] van Rhijn BW, Burger M, Lotan Y, et al. Recurrence and progression of disease in non-muscle-invasive bladder cancer: From epidemiology to treatment strategy. Eur Urol 2009;56:430-42.

[6] Greene FL, Gospodarowicz M, Wittekend C. American Joint Committee on Cancer (AJCC) staging manual. 7th ed. Philadelphia, PA: Springer; 2009.

[7] Linton KD, Rosario DJ, Thomas F, et al. Disease specific mortality in patients with low risk bladder cancer and the impact of cystoscopic surveillance. J Urol 2013;189:828-33.

[8] Bertz S, Otto W, Denzinger S, et al. Combination of CK20 and $\mathrm{Ki}-67$ immunostaining analysis predicts recurrence, progression, and cancer-specific survival in pT1 urothelial bladder cancer. Eur Urol 2014;65:218-26.

[9] Otto W, Denzinger S, Fritsche HM, et al. Introduction and first clinical application of a simplified immunohistochemical validation system confirms prognostic impact of Ki-67 and CK20 for stage T1 urothelial bladder carcinoma: Singlecenter analysis of eight biomarkers in a series of three hundred six patients. Clin Genitourin Cancer 2013;11:53744.

[10] Shariat SF, Bolenz C, Godoy G, et al. Predictive value of combined immunohistochemical markers in patients with pT1 urothelial carcinoma at radical cystectomy. J Urol 2009; 182:78-84.

[11] Shariat SF, Passoni N, Bagrodia A, et al. Prospective evaluation of a preoperative biomarker panel for prediction of upstaging at radical cystectomy. BJU Int 2014;113:70-6.

[12] van Oers JM, Wild PJ, Burger M, et al. FGFR3 mutations and anormal CK20 staining pattern define low-grade noninvasive urothelial bladder tumours. Eur Urol 2007;52:760-8.

[13] Lopez-Beltran A, Luque RJ, Alvarez-Kindelan J, et al. Prognostic factors in survival of patients with stage Ta and T1 bladder urothelial tumors: The role of G1-S modulators (p53, p21Waf1, p27Kip1, cyclin D1, and cyclin D3), proliferation index, and clinicopathologic parameters. Am J Clin Pathol 2004; 122:444-52.

[14] Wu TT, Chen JH, Lee YH, et al. The role of bcl-2, p53, and ki-67 index in predicting tumor recurrence for low grade superficial transitional cell bladder carcinoma. J Urol 2000;163(3):758-60. 
[15] Lotan Y, Bagrodia A, Passoni N, et al. Prospective evaluation of a molecular marker panel for prediction of recurrence and cancer-specific survival after radical cystectomy. Eur Urol 2013;64:465-71.

[16] Krabbe LM, Lotan Y, Bagrodia A, et al. Prospective comparison of molecular signatures in urothelial cancer of the bladder and the upper urinary tract - is there evidence for discordant biology? J Urol 2013. pii: S0022-5347(13)05470-0. doi: 10.1016/j.juro.2013.09.031. [Epub ahead of print]

[17] Margulis V, Shariat SF, Ashfaq R, et al. Ki-67 is an independent predictor of bladder cancer outcome in patients treated with radical cystectomy for organ-confined disease. Clin Cancer Res 2006;12:7369-73.

[18] van Rhijn BW, Vis AN, van der Kwast TH, et al. Molecular grading of urothelial cell carcinoma with fibroblast growth factor receptor 3 and MIB-1 is superior to pathologic grade for the prediction of clinical outcome. J Clin Oncol 2003;21:1912-21.

[19] van Rhijn BW, Zuiverloon TC, Vis AN, et al. Molecular grade (FGFR3/MIB-1) and EORTC risk scores are predictive in primary non-muscle-invasive bladder cancer. Eur Urol 2010;58:433-41.

[20] Harnden P, Mahmood N, Southgate J. Expression of cytokeratin 20 redefines urothelial papillomas of the bladder. Lancet 1999;353:974-7.
[21] Rieken M, Xylinas E, Kluth L, et al. Long-term cancerspecific outcomes of TaG1 urothelial carcinoma of the bladder. Eur Urol 2014;65:201-9.

[22] Holmang S, Hedelin H, Anderstrom C, et al. Recurrence and progression in low grade papillary urothelial tumors. J Urol 1999;162:702-7.

[23] Holmang S, Andius P, Hedelin H, Wester K, Busch C, Johansson SL. Stage progression in Ta papillary urothelial tumors: Relationship to grade, immunohistochemical expression of tumor markers, mitotic frequency and DNA ploidy. J Urol 2001;165(4):1124-8; discussion 1128-30.

[24] Gontero P, Casetta G, Zitella A, Ballario R, Pacchioni D, Magnani C, Muir GH, Tizzani A. Evaluation of P53 protein overexpression, Ki67 proliferative activity and mitotic index as markers of tumour recurrence in superficial transitional cell carcinoma of the bladder. Eur Urol 2000;38(3):287-96.

[25] Quintero A, Alvarez-Kindelan J, Luque RJ, GonzalezCampora R, Requena MJ, Montironi R, Lopez-Beltran A. Ki-67 MIB1 labelling index and the prognosis of primary TaT1 urothelial cell carcinoma of the bladder. J Clin Pathol 2006;59(1):83-8.

[26] Blandamura S, D'Alessandro E, Giacomelli L, Guzzardo V, Battanello W, Repele M, Ninfo V. Expression of maspin in papillary $\mathrm{Ta} / \mathrm{T} 1$ bladder neoplasms. Anticancer Res 2008;28(1B):471-8. 